\title{
EXPERIENCIA EN LA UTILIZACIÓN DE LA PINZA ELECTROQUI- RÚRGICA DE COAGULACIÓN BIPOLAR PLASMACINÉTICA EN LA HISTERECTOMÍA VAGINAL EN EL HOSPITAL CENTRAL NORTE DE PEMEX
}

\author{
Víctor Vázquez Z. ${ }^{1}$, Jorge Zepeda Z. ${ }^{1}$, Carlos Briones L. ${ }^{1}$, Mariana Hernández R. ${ }^{1}$ \\ 1 Servicio de Ginecología, Hospital Central Norte PEMEX, México, DF.
}

\section{RESUMEN}

Objetivo: Describir la morbilidad, pérdida sanguínea y tiempo quirúrgico en la realización de histerectomía vaginal con el uso de la pinza electroquirúrgica de coagulación bipolar plasmacinética. Metodología: Se realizó un estudio retrospectivo en 18 pacientes sometidas a histerectomía vaginal por patología benigna uterina, utilizando energía plasmacinética durante el período de enero de 2007 a febrero de 2009, en el Hospital Central Norte de Petróleos Mexicanos. Resultados: Tiempo quirúrgico de 59,4 \pm 19,3 min (rango: 50-85 $\mathrm{min}$ ), sangrado posoperatorio de 76,6 $\pm 22,3 \mathrm{ml}$ (rango: $30-110 \mathrm{ml}$ ), peso del útero $497,3 \pm 88,9 \mathrm{~g}$ (rango: $245-635 \mathrm{~g}$ ) y tiempo de estancia intrahospitalaria de 2,1 \pm 0,6 días (rango: 1-3 días). No se reportaron complicaciones durante el procedimiento ni en su recuperación posterior. Conclusiones: El uso de la pinza electroquirúrgica de coagulación bipolar plasmacinética, es una alternativa efectiva y segura durante la histerectomía vaginal. Es necesaria la realización de un ensayo aleatorizado para compararla con la técnica tradicional para demostrar otros beneficios.

\section{PALABRAS CLAVE: Histerectomía vaginal, pinza electroquirúrgica de coagulación bipolar}

\section{SUMMARY}

Objective: To describe morbility, blood loss and procedure time of vaginal hysterectomy using an electrosurgical bipolar vessel sealer. Methods: Patients scheduled for vaginal hysterectomy using electrosurgical bipolar vessel sealer as the hemostasis technique during the period January 2007 to February 2009, at the Central Hospital North of Petroleos Mexicanos. Results: A total of 18 patients underwent vaginal hysterectomy for some benign disease of the uterus. Among these patients, mean procedure time in the electrosurgical bipolar vessel sealer was $59.4 \pm 19.3 \mathrm{~min}$ (range: $50-85 \mathrm{~min}$ ). Mean estimated blood loss was $76.6 \pm$ $22.3 \mathrm{ml}$ (range: $30-110 \mathrm{ml}$ ), weight of the uterus was $497.3 \pm 88.9 \mathrm{~g}$ (range: $245-635 \mathrm{~g}$ ), and length of stay $2.1 \pm 0.6$ days (range: $1-3$ days). There was no perioperative complication. Conclusion: Electrosurgical bipolar vessel sealer is an effective alternative to sutures in vaginal hysterectomy. Larger adequately-powered studies are however still required.

KEY WORDS: Vaginal hysterectomy, electrosurgical bipolar vessel sealer 


\section{INTRODUCCIÓN}

La histerectomía es una de las cirugías más frecuentemente realizadas en el mundo. En Estados Unidos se realizan aproximadamente 600.000 histerectomías cada año, siendo superada sólo por la cesárea $(1,2,3)$. La vía más usada es la abdominal y la de menor prevalencia de complicaciones es la vaginal $(1,2)$.

La electrocirugía en nuestros días comprende una parte fundamental de la cirugía, siendo una herramienta para favorecer el corte y la coagulación durante los procedimientos y es de suma importancia conocerla en detalle para así poder disminuir el riesgo de accidentes y darle un uso óptimo (4).

La pinza electroquirúrgica de coagulación bipolar plasmacinética tiene un rendimiento consistente a través de un amplio rango de impedancias en el tejido, formando bolsas de vapor en tejidos de baja impedancia $(4,5)$ y así provocando la coagulación. Tiene el mejor rendimiento cuando el tejido se humedece, no necesita placa y permite realizar una hemostasia consistente sin adherirse (4). Forma un sello de manera efectiva que coagula vasos de hasta $7 \mathrm{~mm}$ de diámetro y mantiene una presión de $300 \mathrm{mmHg}$ (4).

En el presente estudio se decidió examinar el tiempo quirúrgico, el sangrado quirúrgico, el peso uterino y la prevalencia de complicaciones en pacientes en quienes se usó la pinza electroquirúrgica de coagulación bipolar plasmacinética (Plasmakinetics) al realizar la histerectomía vaginal.

\section{PACIENTES Y MÉTODOS}

Se realizó un estudio retrospectivo, a través de la revisión de expedientes clínicos del servicio de ginecología del Hospital Central Norte de Petróleos Mexicanos, en quienes se realizó histerectomía vaginal por indicación benigna, con el uso de la pinza electroquirúrgica de coagulación bipolar plasmacinética (PlasmaKinetics ${ }^{\mathrm{TM}}$ sealer, Gyrus Medical, Maple Grove, Minnesota) (Figura 1), durante el período de enero de 2007 a febrero de 2009. El estudio fue aprobado por el comité de ética del Hospital Central Norte, $y$ todas las pacientes que ingresaron contaban con el antecedente de acceder al procedimiento a través de un consentimiento informado.

Serie de casos consecutivos, en que cada paciente fue ingresada en una base de datos de Excel previo a la cirugía, creando campos con distintas variables epidemiológicas, cuantitativas y cualitativas. Posteriormente se analizaron variables relacionadas al procedimiento quirúrgico, como tiempo operatorio, cantidad de sangrado, peso del útero y complicaciones durante el procedimiento y en el postoperatorio mediato y tardío. Los procedimientos fueron realizados por un solo cirujano.

Técnica quirúrgica (Figura 2): Paciente en posición de litotomía, tracción del cérvix para incidir circuncidándolo, y posteriormente disección manual roma de la vejiga, hasta que es separada del útero y se corta el peritoneo anterior. Se disecó la fascia vaginal posterior hasta abrir el fondo de saco. El útero fué extirpado en 3 pasos, en homología a la técnica tradicional, pues la pinza de Plasmakinetics se usa de la misma forma que las pinzas Heaney durante la técnica estándar y se diferencian en tener un electrodo bipolar en la parte interna de la superficie prensil. La hemostasia con suturas es sustituida al colocar el fórceps abierto y producir el sello seguido por el corte, se coagularon y cortaron los ligamentos uterosacros, seguido de los pedículos de los ligamentos cardinales y de los vasos uterinos. Se palpan los ovarios, y si se van a conservar (como en todos nuestros casos), se realiza el mismo procedimiento para los ligamentos utero-ováricos. Se verifica la hemostasia y la cúpula vaginal fue cerrada con sutura continua de catgut crómico 0 . El tiempo del procedimiento se midió desde la incisión en el cérvix hasta el cierre completo de la cúpula vaginal. Las pérdidas sanguíneas se estimaron por el servicio de anestesiología, midiendo lo recolectado en los colectores de aspiración y la cantidad de compresas y gasas utilizadas. El útero fue pesado inmediatamente, por el personal de enfermería, a la extracción en la báscula de quirófano. Otras variables fueron tiempo de estancia hospitalaria, medido desde el inicio del día que se realizo la cirugía, hasta el egreso de la paciente del servicio. Las complicaciones postoperatorias se registraron en las visitas de seguimiento a la semana y a las 4 semanas posteriores a la cirugía.

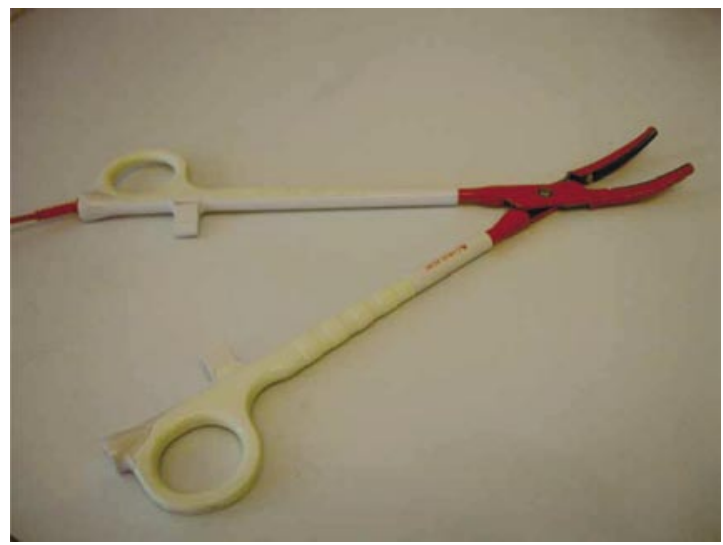

Figura 1. Pinza electroquirúrgica de coagulación bipolar plasmacinética (PlasmaKinetics ${ }^{\mathrm{TM}}$ sealer, Gyrus Medical, Maple Grove, Minnesota). 


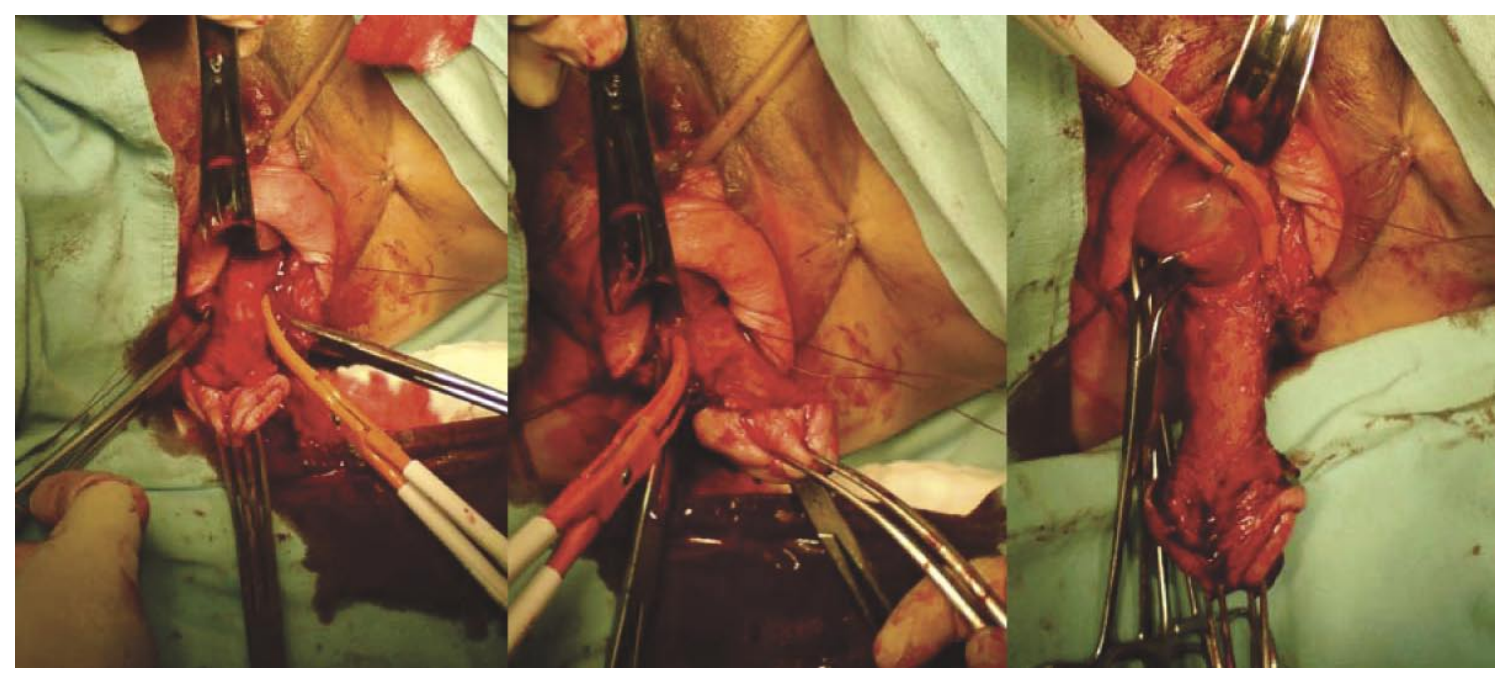

Figura 2. Uso de la pinza electroquirúrgica de coagulación bipolar plasmacinética en histerectomía vaginal.

Las distintas variables fueron analizadas mediante el programa estadístico SSPS versión 17, con el que obtuvimos promedio, desviación estándar y rango.

\section{RESULTADOS}

Se realizaron un total de 18 histerectomías vaginales. Las principales indicaciones fueron por prolapso uterino $66,6 \%$, sangrado uterino anormal $16,6 \%$ y miomatosis uterina en el $16,6 \%$. La edad promedio de las pacientes fue $65,6 \pm 7,7$ años (rango: 59-80 años), con un índice de masa corporal (IMC) 29,8 \pm 3 (rango: 26-34).

El tiempo quirúrgico fue de 59,4 $\pm 19,3$ min (rango: $50-85 \mathrm{~min}$ ). Sangrado postoperatorio de 76,6 $\pm 22,3 \mathrm{ml}$ (rango: $30-110 \mathrm{ml}$ ). Tiempo de estancia intrahospitalaria fue de 2,1 $\pm 0,6$ día (rango: 1-3 días). El peso uterino tuvo una media de 497,3 \pm 88,9 gramos (rango: $245-635 \mathrm{~g}$ ). No se reportaron complicaciones durante el procedimiento, ni en su recuperación posterior, hasta la cuarta semana de seguimiento.

\section{DISCUSIÓN}

En los últimos años, debido a la aparición de nuevas técnicas hemostáticas, es necesario medir los efectos médicos y económicos de las distintas alternativas quirúrgicas existentes para la resección uterina (6). El optar por la vía vaginal ha demostrado beneficios en ambas áreas; pero es importante considerar diversos factores como el tamaño uterino, la patología con compromiso extrauterino, la severidad del diagnóstico y lo más importante, la existencia de preparación y experiencia de los especialistas para realizar la cirugía elegida (7).

En la mayoría de los reportes internacionales, durante un procedimiento quirúrgico mayor, el uso de plasmakinetics reduce el tiempo quirúrgico, la hemorragia y la estancia intrahospitalaria $(4,7)$, variables que impactan directamente en el costo total del procedimiento a nivel institucional $(2,7)$. Su uso también favorece el abordaje, facilitando el procedimiento para cirujanos menos experimentados, o en el caso de pacientes con obesidad mórbida o comorbilidad (8), por lo que esta tecnología podría estandarizarse para disminuir la incidencia de complicaciones en pacientes técnicamente difíciles.

Una de las ventajas importantes, como lo refirieron Dessole y cols (9), fue la reducción de la salida fortuita de corriente eléctrica, así realzando la seguridad para el paciente y el cirujano. Con respecto a la electrocirugía monopolar, el electrocauterio bipolar es más controlable (9).

En el estudio prospectivo de Candiani y cols (10), refirieron que el tiempo medio con técnica estándar durante la histerectomía vaginal por indicación benigna fué de $81 \pm 30$ minutos, con hemorragia de $178 \pm 149 \mathrm{ml}$ y promedio de estancia de 3,2 $\pm 0,6$ días (10). Estos resultados y los de otros autores muestran que el uso de instrumentos electroquirúrgicos podría disminuir el tiempo operatorio, la hemorragia y los días de estancia intrahospitalaria, comparado con la técnica tradicional $(4,10)$.

Durante nuestra serie de casos, el uso de plasmakinetics durante la histerectomía vaginal, demostró un tiempo quirúrgico aceptable, con volúmenes 
de sangrado transoperatorio menores a la hemorragia esperada (10), por lo que es posible inferir de que se podrían disminuir las indicaciones de transfusión de hemoderivados y los riesgos asociados. La estancia hospitalaria se acortó con el uso de la pinza, comparado con el promedio de otros autores que usaron cirugía tradicional (10), por lo que las pacientes pueden reincorporarse de forma más breve a sus actividades cotidianas, resultando en un posible impacto benéfico en costos por permanencia hospitalaria e incapacidades laborales.

Adicionalmente, las pacientes deberán ser instruidas acerca de la convalecencia, dolor postoperatorio y complicaciones asociadas al abordaje vaginal y el uso de esta tecnología. La educación del paciente facilitará el éxito de la vía vaginal con la energía bipolar $(4,10)$.

Nuestra experiencia con esta modalidad, como todas, cuenta con puntos a favor y debilidades, sin embargo, en nuestra población se ha demostrado evolución favorable. Será necesario realizar otro estudio, idealmente un ensayo clínico aleatorizado para evaluar un mayor número de variables trans y postquirúrgicas (incluyendo dolor y comparación de costos) para evaluarlo contra la técnica tradicional, así como la evaluación de la implementación para otras cirugías ginecológicas.

El abordaje quirúrgico para la histerectomía, deberá ser decidido por el médico y la paciente, orientando a las mujeres de forma clara y completa, para decidir de acuerdo a sus necesidades, deseos y expectativas, la vía e instrumentos adecuados para cada una de ellas.

\section{CONCLUSIÓN}

El uso de la pinza electroquirúrgica de coagulación bipolar plasmacinética, es una alternativa efectiva y segura durante la histerectomía vaginal. Es necesaria la realización de un ensayo aleatorizado para compararla con la técnica tradicional para demostrar otros beneficios.

\section{BIBLIOGRAFÍA}

1. Murillo JM, Pedraza LA, Aguirre X, López PE. Histerectomía por laparoscopia: Experiencia de 10 años en el Hospital Español de México. Ginecol Obstet Mex 2007; 75(11):667-77.

2. Nezhat C, Nezhat F, Nezhat C. Nezhat's Operative Gynecologic Laparoscopy and Hysteroscopy; $3^{\mathrm{a}}$ Ed. Cambridge University Press 2008;13:341-62.

3. Farqhuar C, Steiner C. Hysterectomy Rates in the United States 1990-1997. Obstet Gynecol 2002;99: 22934.

4. Presthus JB, Brooks PG, Kirchhof N. Vessel sealing using a pulsed bipolar system and open forceps. J Am Assoc Gynecol Laparosc 2003;10(4):528-33.

5. Levie MD, Chudnoff SG. Energy Sources for Endoscopic Surgery. Abstracts for the Global Congress of Gynecologic Endoscopy. 33rd annual meeting of the AAGL. August 2004, Vol. 11 No.3 Supplement.

6. Salinas H, Pasten J, Naranjo B, Carmona S, Retamales B, Díaz G, Franulic L. Análisis clínico y económico de la histerectomía abdominal versus la histerectomía vaginal en el Hospital Clínico de la Universidad de Chile. Revisión de 2.338 casos. Rev Chil Obstet Ginecol 2006;71(4):227-33.

7. Johnson N, Barlow D, Lethaby A, Tavender E, Curr L, Garry R. Methods of hysterectomy: systematic review and meta-analysis of randomized controlled trial. BMJ 2005;330(7506):1478.

8. Hagen B, Eriksson N, Sundset M. Randomised controlled trial of LigaSure versus conventional suture ligature for abdominal hysterectomy. BJOG 2005;112(7):968-70.

9. Dessole S, Rubattu G, Capobianco G, Caredda S, Cherchi PL. Utility of bipolar electrocautery scissors for abdominal hysterectomy. Am J Obstet Gynecol. 2000;183(2):396-9.

10. Candiani M, Izzo S, Bulfoni A, Riparini J, Ronzoni S, Marconi A. Laparoscopic vs vaginal hysterectomy for benign pathology. Am J Obstet Gynecol 2009;200(4):368.e1-7. 